\title{
A New Method for Detecting Cerebral Tissues Abnormality in Magnetic Resonance Images
}

\author{
Mohammed Sabbih Hamoud Al-Tamimi ${ }^{1,2} \&$ Ghazali Sulong ${ }^{1}$ \\ ${ }^{1}$ UTM-IRDA Digital Media Centre (MaGIC-X), Faculty of Computing, Universiti Teknologi Malaysia, Malaysia \\ ${ }^{2}$ Department of Higher Studies, University of Baghdad, Al-Jaderia, Baghdad, Iraq \\ Correspondence: Ghazali Sulong, UTM-IRDA Digital Media Centre (MaGIC-X), Faculty of Computing, \\ Universiti Teknologi Malaysia, 81310 Skudai, Johor Bahru, Malaysia. Tel: 60-177-467-128. E-mail: \\ ghazali@utmspace.edu.my/m_altamimi75@yahoo.com
}

Received: December 9, 2014

Accepted: January 14, 2015

Online Published: July 30, 2015

doi:10.5539/mas.v9n8p354

URL: http://dx.doi.org/10.5539/mas.v9n8p354

\begin{abstract}
We propose a new method for detecting the abnormality in cerebral tissues present within Magnetic Resonance Images (MRI). Present classifier is comprised of cerebral tissue extraction, image division into angular and distance span vectors, acquirement of four features for each portion and classification to ascertain the abnormality location. The threshold value and region of interest are discerned using operator input and Otsu algorithm. Novel brain slices image division is introduced via angular and distance span vectors of sizes $24^{\circ}$ with 15 pixels. Rotation invariance of the angular span vector is determined. An automatic image categorization into normal and abnormal brain tissues is performed using Support Vector Machine (SVM). Standard Deviation, Mean, Energy and Entropy are extorted using the histogram approach for each merger space. These features are found to be higher in occurrence in the tumor region than the non-tumor one. MRI scans of the five brains with 60 slices from each are utilized for testing the proposed method's authenticity. These brain images (230 slices as normal and 70 abnormal) are accessed from the Internet Brain Segmentation Repository (IBSR) dataset. 60\% images for training and $40 \%$ for testing phase are used. Average classification accuracy as much as $98.02 \%$ (training) and 98.19\% (testing) are achieved.
\end{abstract}

Keywords: abnormal brain tissues, brain slices images, cerebral tissues, image classification, magnetic resonance imaging, statistical features.

\section{Introduction}

Lately, MRI is employed in diverse medical fields, including heart diseases, cancer research and brain diseases (El-Dahshan et al., 2009). It creates high-quality two or three dimensional images of an object to accurately visualize and detect the brain tumors.

Furthermore, the images provide valuable and detailed information regarding normal and abnormal tissues. Brain tumor being the most common and deadly brain diseases can affect and devastate many lives. According to International Agency for Research on Cancer (IARC) report, over 126000 people having brain tumor are diagnosed worldwide per year with more than 97000 mortality rate (Al-Tamimi \& Sulong, 2014b; Ferlay et al., 2010). At present, MRI is the most common test for diagnosing and confirming the presence of brain tumor. It identifies the tumor location for recommended specialist treatment options (Horská \& Barker 2010; Al-Tamimi \& Sulong, 2014b; Al-Tamimi \& Sulong, 2014a).

The diagnostic flaws needs considerable reduction through precise diagnosis and detection of the brain abnormality related to the type, size and effected area to aid appropriate treatment planning. Computer Aided Diagnosis (CAD) systems are introduced to enhance the diagnostic accuracies (Roy et al., 2013; Al-Tamimi \& Sulong, 2014b; Al-Tamimi \& Sulong, 2014a). In fact, provides CAD output of the computer system second opinion to help the radiologists image interpretations by considerably reducing the image reading time. These computer based auto detection systems often help in improve the accuracy of radiation analyses (Selvanayaki \& Karnan, 2010).

In the medical field, image processing and analysis is of great significance, particularly in noninvasive treatment and clinical study (Spisz \& Bankman, 2000). Medical image processing emerged as one of important and 
reliable methods in identifying and diagnosing different tissues disorders and abnormalities. Imaging diagnostics assist the doctors/radiologists to visualize, analyze and understand the nature of abnormalities in internal organ structures for specific diagnosis and treatment (Veloz et al., 2011). MRI possessing notable advantages of non-invasiveness and soft tissue contrast remains one of the widely-used techniques for brain imaging (Gang et al., 2013; Sikka et al., 2009).

Undoubtedly, the brain being the command centre is the key organ responsible for controlling diverse functions related to body movement, blood flow, cardiac activities, temperature of the body, emotional behaviour, learning and memorization, to cite a few. Despite intensive research, the classification of MRI data in terms of normal and abnormal brain tissues for performing fast and accurate diagnostics remains challenging. Segmentation of brain for abnormality detection in slice image is the most a daunting task due to its complex anatomy and problems inherent to the nature of the image. The heterogeneous and diffuse manifestation of pathology in medical images often prohibits the employment of computational methods. Furthermore, all imaging modalities carry limitations and artifacts which must be addressed and solved by segmentation methods. In this study the brain images are selected for the image reference as the injuries or abnormalities which can affect the large part of an organ.

Formerly, different methods used to classify the MRI data are divided into two categories. First one includes the supervised learning techniques such as SVM (Salankar \& Bora, 2014), Artificial Neural Network (ANN) (Joshi et al., 2010; Ibrahim et al., 2013) and K-Nearest Neighbor (KNN) (Al-Badarneh et al., 2012; Rajini \& Bhavani, 2011; Badran et al., 2010). The other one containing unsupervised learning methods as Self-Organizing Map (SOM) (Goswami \& Bhaiya, 2013), k-means Clustering (Deepak et al., 2013), Statistical Images and Analysis (SIA) (Technologies, 2014) are mainly used for data clustering.

We use SVM to classify MR images based on normal and abnormal brain tissues. The proposed new method combines four steps such as extraction of the cerebral tissues, their classification into angular and distance spans, determination of four features for each portion and identification of the abnormality location. A Brain slice image division using angular and distance span is introduced by moving one portion of the vector entries clockwise. Rotation invariance of the angular span vectors of the sizes $24^{\circ}$ and 15 pixels is applied. The results are analysed, compared and understood.

\section{Brain Anatomy}

The human brain is the command centre that controls all body parts and allows in adapting varying environmental conditions. The brain as displayed in Figure 1 is composed of two types of tissues called Gray Matter (GM) and White Matter (WM). GM consists of neuronal and glial cells often termed as neuroglia or glia which controls brain activity. The basal nuclei called GM nuclei are located deep within the WM. The basal nuclei contain caudate nucleus, putamen, pallidum and claustrum. WM fibers consist of elinated axons which connect the cerebral cortex to other parts of the brain. The left and the right hemispheres of the brain are connected by the corpus callosum, which is a thick band of WM fibres (Noback et al., 2005; Al-Tamimi \& Sulong, 2014b).

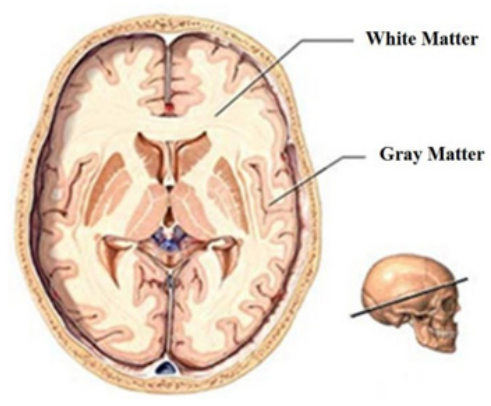

Figure 1. Grey Matter and White Matter Tissues (Nolte, 2013)

The Cerebrospinal Fluid (CSF) in the brain as shown in Figure 2 is comprised of enzymes, glucose, salts and white blood cells. Cerebrospinal fluid circulates through ventricles around the brain and the spinal cord to protect from injuries. Another type of tissues called meninges that present in form of membrane to cover the brain and spinal cord (Noback et al., 2005; Al-Tamimi \& Sulong, 2014b). 


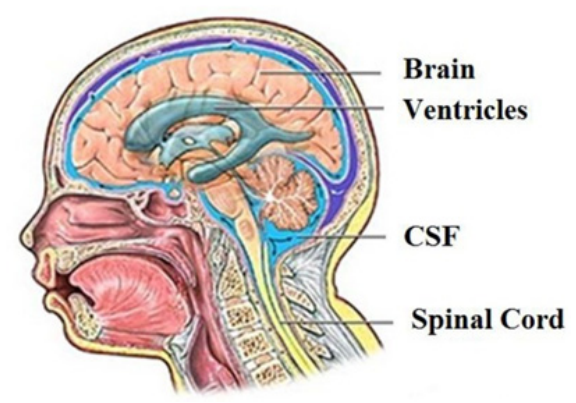

Figure 2. Normal circulation of CSF in the brain (Nolte 2013)

Figure 3 illustrates the brain anatomy which consists of brain stem and cerebrum. The cerebrum inhibits the largest part of the brain and responsible for the movement, conscious thoughts and sensations. The cerebrum is divided into left and the right hemispheres. Each cerebrum hemisphere controls the opposite side of the body and each half is further partitioned into four parts including frontal, temporal, parietal and occipital lobes. The cerebellum being the second largest structure of the brain is connected to the controlling motor functions of the body, such as balance, walking, posture and the general motor coordination. It is located at the rear side of the brain and attached to the brain stems. Both, cerebrum and cerebellum possess ultra-thin outer cortex of gray matter, internal white matter and small but deeply situated masses of the gray matter. The spinal cord is joined to the brainstem and positioned towards the brain bottom. The brainstem controls various fundamental functions such as motor, sensory pathways, cardiac, repository and reflexes. It is divided into the midbrain, pons and medulla oblongata membrane (Noback et al., 2005; Al-Tamimi \& Sulong, 2014b).

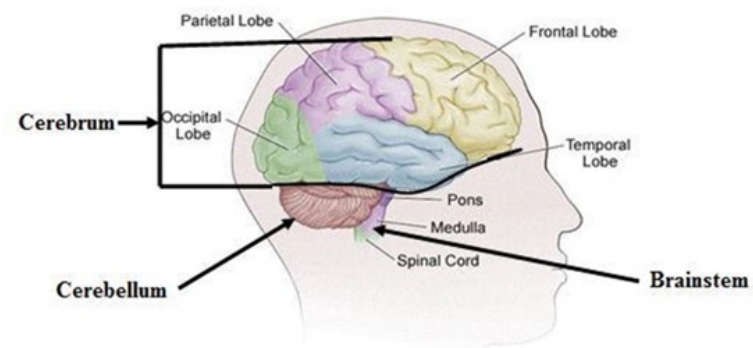

Figure 3. Major subdivisions of the human brain (Nolte 2013).

\section{Related Work}

Radiologists analyze the MR images by visual inspection to detect and determine if a tumor or abnormal tissue. The huge number of such images makes this visual interpretation process expensive and often erroneous. Furthermore, the sensitivity of the human eye and brain to elucidate such images reduces with the increase of number of cases, especially when only a small number of slices contain information of the affected area. Therefore, an automated system for analysis and classification MR images is essential.

Practically, MR images include both normal and defective slices. Firstly, these defective or abnormal slices are detected and separated from the normal slices. Secondly, these abnormal slices are further examined to identify the exact nature and location of tumors. Following slice segmentation with fuzzy c-means (FCM) algorithm, Clarke et al., (Clark et al., 1994) identified the abnormality in MR images. Antonie (Antonie, 2008) introduced a method for automated segmentation and categorization of brain MR images using SVM classifier. The normal and abnormal slices are identified based on statistical features. However, recent investigations in data classification reveal that Least Squares Support Vector Machines (LS-SVMs) approach is highly capable of providing higher sorting accuracy compared to existing algorithms (Cawley \& Talbot, 2004; Improved et al., 2005).

The likelihood of detecting a premature dementia without using rigid registration of MRI is established (Klein et al., 2010). Based on the dissimilarity matrix, a k-nearest neighbors (k-NN) classifier is developed. The efficiency and performance of the classifier are tested in a leave-one-out experiment on 58 images. This method achieves 
an efficiency of $81 \%$. Hybrid techniques consisting of three steps including feature extraction via Discrete Wavelet Transform (DWT), reduce the dimensions size by Principal Component Analysis (PCA) and classification of the outputs using two classifiers are proposed (El-Dahshan et al., 2010). The classifiers are based on ANN and k-NN. The dataset comprised of T2-weighted, axial dimension, $256 \times 256$ pixels, image size 70 (with 10 normal and 60 abnormal) are employed. Remarkably, the number of extracted features is reduced from 1024 to seven using PCA. Accuracy as much as $97 \%$ and $98 \%$ are achieved from DWT+PCA+ANN and $\mathrm{DWT}+\mathrm{PCA}+\mathrm{k}-\mathrm{NN}$, respectively.

In the past, MR brain images are classified using ANN and SVM method (Chaplot et al., 2006). The pre-processing phase involving DWT is used as input for Neural Network (NN) and SVM. The dataset consisting of T2-weighted, axial, $256 \times 256$ pixels MRI, images size 52 with 46 for abnormal (marked by Alzheimer's disease) and 6 for normal are applied, where 4761 features are extracted. The achieved accuracy of the classifier DWT+SOM is $94 \%$, DWT+SVM with linear kernel is $96.15 \%$, DWT+SVM with polynomial kernel is $98.00 \%$ and DWT+SVM with radial basis function based kernel is $98.00 \%$. An automatic classification of MR images for normal or abnormal tissues is proposed (Ariffanan \& Basri, 2008). This classifier follows two steps such as feature extraction by PCA and classification by the neuro fuzzy. Using an input dataset of size 35 (with 20 as training set and 15 as testing set) the accuracy of $93.33 \%$ is achieved.

\section{Methodology}

Our proposed method comprised of four steps, including the extraction of cerebral tissues, division of cerebral tissues into angular and distance span, attainment of four features for each portion and finally the use of classification to detect the abnormality location. Figure 4 represents the flowchart of the proposed method. The slice image of MR representations is used to extract the brain tissues from non-brain one by skull stripping. Threshold value is automatically selected via Otsu's algorithm (Otsu, 1979). Following angular and distance span, new brain slice image division is introduced. The divided brain MR images are employed as input in the feature extraction algorithm. Four statistical features are calculated for each merger space. The features pattern vectors obtained from the divided images are utilized as input of SVM classifier. Five brain MR images with each image containing 60 slices are used for testing and validation. Total of 230 slices are considered as normal brain images and rest 70 slices are taken as abnormal brain images. Sixty percent MR brain slice images are utilized for the training phase and remaining $40 \%$ are used for the testing phase.

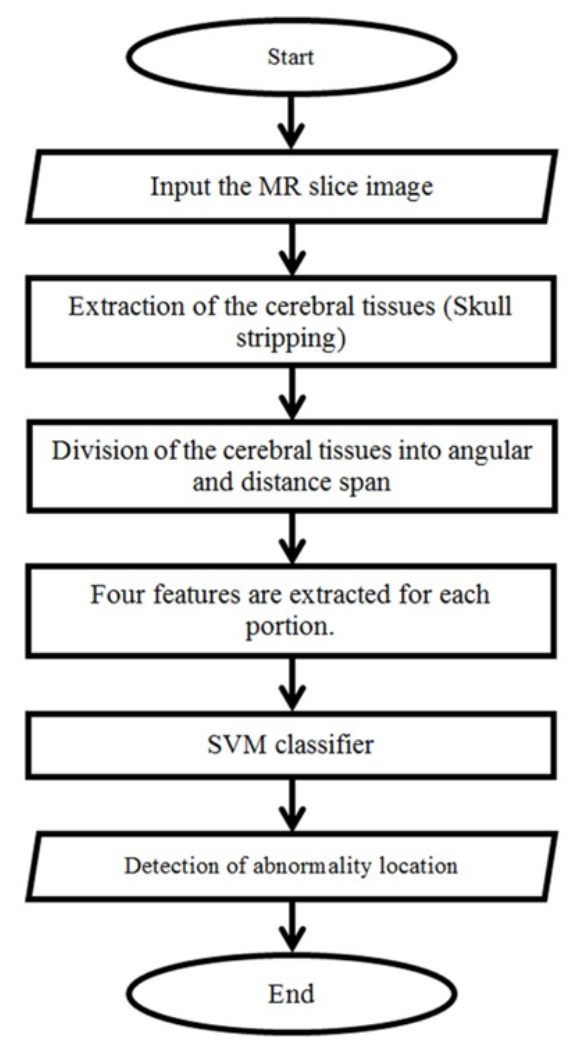

Figure 4. Flowchart of the proposed method. 


\subsection{Skull Stripping}

Skull stripping is one of the significant phases in neurology, where brain tissues are extracted from non-brain tissues within MR images. Several skull stripping methods are developed in the past (Zhang et al., 2011; Sadananthan et al., 2010; Ségonne et al., 2004). Skull stripping algorithms are generally categorized into four groups based on morphological, deformable surface, atlas and hybrid nature (Ségonne et al., 2004). In usual practice the skull stripping is performed using threshold intensity (Ségonne et al., 2004; Sadananthan et al., 2010).

The proposed skull stripping method consisting of three steps is shown in Figure 5. Firstly, the image binarization is performed using threshold value via Otsu's automatic selection algorithm. Secondly, the largest connected portion of the binarized image is chosen, assuming skull as one of the major parts surrounding the head. Thirdly, the skull stripped brain image is obtained after extracting the cerebral tissues.

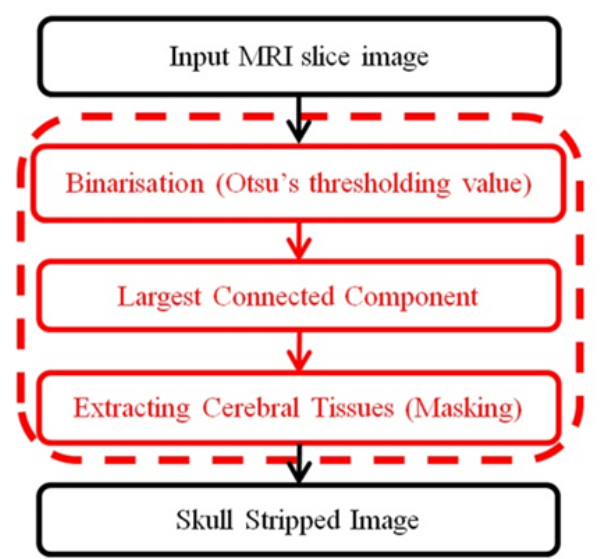

Figure 5. Steps of skull stripping.

\subsubsection{Binarization}

Image binarzation is the simplest method for image segmentation that transforms an image gray level into two values only. Selection of optimum threshold value " $K$ " ensures the separation of MR image into the background (formed by the very low intensity pixels as part of CSF) and foreground (includes the GM and WM of the brain) tissues as shown in Figure 6.

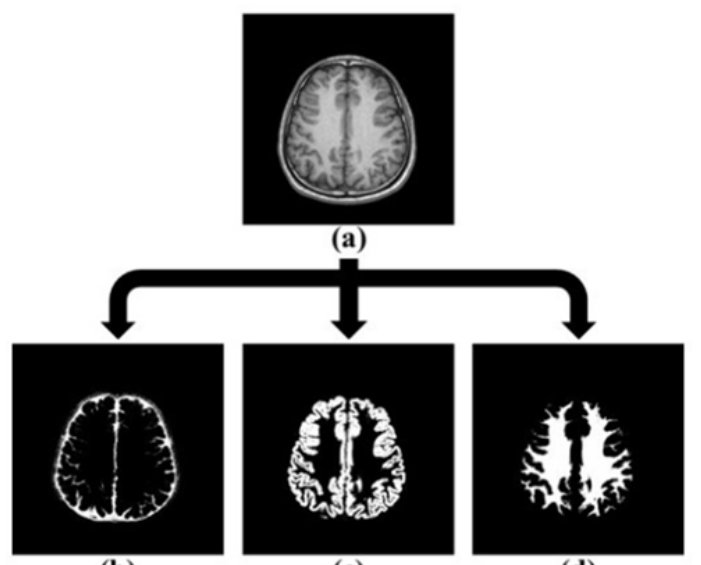

(b)

(c)

(d)

Figure 6. Components of the brain. (a) MRI Slice, (b) segmented CSF, (c) segmented GM and (d) segmented WM (Chuang et al., 2012).

Otsu's method (Otsu, 1979) is employed to obtain cluster-based image threshold value by transforming a gray level image into binary image. It is further assumed that the image comprises of two types of pixels, or bi-model histogram such as the background and the foreground. The optimum threshold values for these two types of pixels are calculated separately to minimize their combined spread (intra-class variance). Multi-level threshold is achieved by extending Otsu's algorithm. The threshold value is carefully selected by reducing the intra-class 
variance (Chen, 2004) which is defined as the weighted sum of variances of two classes (Otsu 1979):

$$
\sigma_{\omega}^{2}(t)=\omega_{1}(t) \sigma_{1}^{2}(t)+\omega_{2}(t) \sigma_{2}^{2}(t)
$$

where $\omega_{1}$ and $\omega_{2}$ are the probabilities of the two classes separated by a threshold $t$ and $\sigma_{1}^{2}$ and $\sigma_{2}^{2}$ are their variances.

Otsu's demonstrated that the process of intra-class variance minimization is equivalent to inter-class variance maximization (Otsu, 1979):

$$
\sigma_{b}^{2}(t)=\sigma^{2}-\sigma_{\omega}^{2}(t)=\omega_{1}(t) \omega_{2}(t)\left[\mu_{1}(t)-\mu_{2}(t)\right]^{2}
$$

Where $\omega_{\mathrm{i}}$ class probabilities and $\mu_{\mathrm{i}}$ are their Mean values. The class probability $\omega_{1}(\mathrm{t})$ is evaluated from the histogram using the expression,

The class Mean $\mu_{1}(t)$ is calculated from,

$$
\omega_{1}(\mathrm{t})=\sum_{0}^{\mathrm{t}} \mathrm{p}(\mathrm{i})
$$

$$
\mu_{1}(\mathrm{t})=\frac{\sum_{0}^{\mathrm{t}} \mathrm{p}(\mathrm{i}) \mathrm{x}(\mathrm{i})}{\omega_{1}}
$$

Where $x(I)$ is the value of the centre $i$ of the histogram bin and $\omega_{2}(t)$ and $\mu_{2}$ on the right-hand side of the histogram can compute for bins larger than $t$. The class probabilities and Means are calculated iteratively using the algorithm.

\subsubsection{Largest Connected Component}

Binarization on brain MR images classifies it into the background and foreground with the foreground into a number of connected components. The component with large area is considered as displayed in Figure 7 with the assumption that the skull is the principal connected structure surrounding the head.

\subsubsection{Extracting Cerebral Tissues (Masking)}

The earlier steps contribute to obtain the binary mask for head MRI scan. The cerebral tissues are extracted by performing bitwise operations between the original head MRI scans with the binary mask Figure 7. In this step, the background noise and other non-brain artifacts are removed.

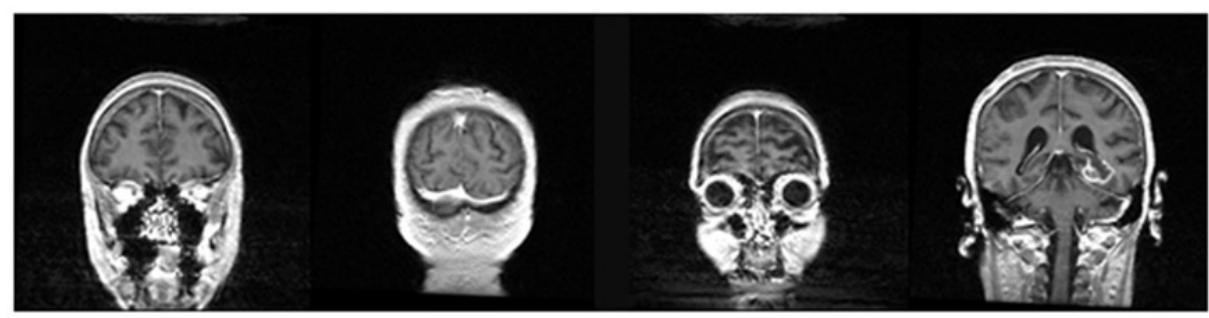

(a)

(b)

(c)

(d)

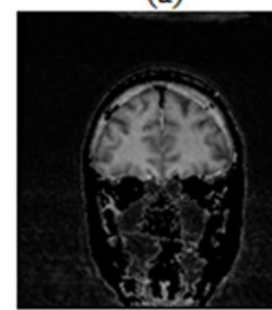

(e)



(f)

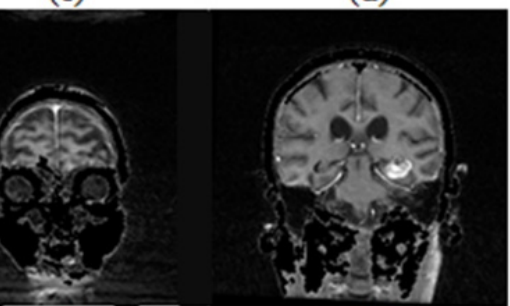

(g)

(h)

Figure 7. Extraction phase with (a), (b), (c) and (d) as the original MR images. Here (e), (f), (g) and (h) are the corresponding cerebral tissues of MR brain images achieved using the proposed method.

\subsection{Gravity Center Point}

The first step in extracting the features of input brain MR slice images is the calculation of the gravity center point on image enabling the proposed method as translational invariant. The Center Of Gravity (COG) method is employed to determine the image features. The coordinates for COG of an MR image $\left(x_{c}, y_{c}\right)$ is determined using, 


$$
\left(\mathrm{x}_{\mathrm{c}}, \mathrm{y}_{\mathrm{c}}\right)=\left(\frac{\sum_{\mathrm{j}=1}^{\mathrm{m}} \sum_{\mathrm{i}=1}^{\mathrm{n}} \mathrm{i}[\mathrm{i}, \mathrm{j}]}{\sum_{\mathrm{j}=1}^{\mathrm{m}} \sum_{\mathrm{i}=1}^{\mathrm{n}} \mathrm{I}[\mathrm{i}, \mathrm{j}]}, \frac{\sum_{\mathrm{j}=1}^{\mathrm{m}} \sum_{\mathrm{i}=1}^{\mathrm{n}} \mathrm{j}[\mathrm{i}, \mathrm{i}]}{\sum_{\mathrm{j}=1}^{\mathrm{m}} \sum_{\mathrm{i}=1}^{\mathrm{n}} \mathrm{I}[\mathrm{i}, \mathrm{j}]}\right)
$$

Where $\mathrm{i}$ is a brain MRI skull stripped slice image of dimension $\mathrm{m} \times \mathrm{n}$ and $\mathrm{x}_{\mathrm{c}}$ and $\mathrm{y}_{\mathrm{c}}$ are the $\mathrm{x}$ and $\mathrm{y}$ coordinates of the brain COG.

\subsection{MRI Slice Image Division}

First two features Figure 7 being very useful for tumor recognition is used to prune the search space during MR image slice determination through the distance and angular span merging division. Subsequently, the rotation invariance of the angular span vector is determined by moving a part of vector entries clockwise. The angular and distance span vectors of sizes $24^{\circ}$ with 15 pixels are used. Figure 8 illustrates the angular and distance span vectors.

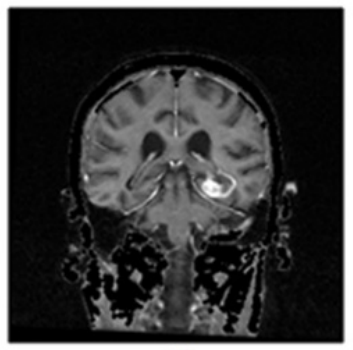

(a)

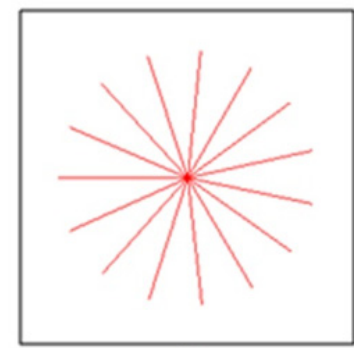

(b)

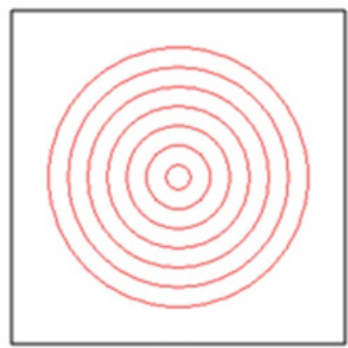

(c)

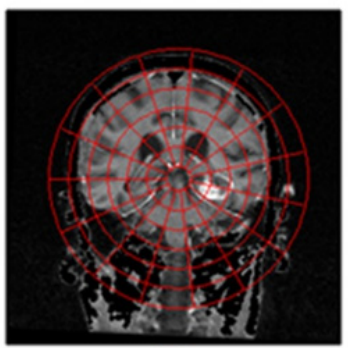

(d)

Figure 8. (a) Cerebral tissues of the brain, (b) Angular span, (c) Distance span and (d) Division of brain MR slice image using these spans.

\subsection{Feature Extraction}

Comparisons are made using different authentic feature extraction methods. They are operated on each division following angular and distance span. The merger between them is applied on the slice image. For each merger space, four statistical features are calculated. Four texture measures are determined from the distributed skull stripped brain slice image assuming that there is no correlation with the neighboring pixel. The texture analysis is performed using intensity value concentrations in the whole or a part of an image represented by a histogram. Histogram approach is followed to extract features including Standard Deviation, Mean, average Energy and Entropy (Jahne, 2005). The histogram intensity levels are simply the summary of the statistical information of the image and particular pixel determining the gray-level. Therefore, the histogram carries the first-order statistical data related to the division space for images. These features are mathematically defined below.

\subsubsection{Mean}

It is an average value which measures the general brightness of an image,

$$
\operatorname{Mean}(\mu)=\frac{\sum_{\mathrm{x}=1}^{\mathrm{M}} \sum_{\mathrm{y}=1}^{\mathrm{N}} \mathrm{I}_{\mathrm{i}}(\mathrm{x}, \mathrm{y})}{\mathrm{M} \times \mathrm{N}}
$$

\subsubsection{Standard Deviation}

It is the measure of the amount of variation or dispersion from the average value. A low value indicates that the data points tend to be very close to the Mean, and a high one implies their spread out over a large range of values. It is expressed as,

$$
\text { Standard Deviation }(\sigma)=\sqrt{\frac{\sum_{x=1}^{M} \sum_{y=1}^{N}(I(x, y)-\mu)^{2}}{M \times N}}
$$

\subsubsection{Energy}

It returns the sum of squared elements of gray level values of all pixels in the image. It is equal to 1 for constant image and given by,

$$
\text { Energy }(\mathrm{e})=\frac{1}{\mathrm{MN}} \sum_{\mathrm{x}=1}^{\mathrm{M}} \sum_{\mathrm{y}=1}^{\mathrm{N}} I^{2}(\mathrm{x}, \mathrm{y})
$$




\subsubsection{Entropy}

It is the measure of non-uniformity in the image based on the probability of the gray level values of all pixels and is defined as,

$$
\text { Entropy }=\frac{1}{\mathrm{MN}} \sum_{\mathrm{x}=1}^{\mathrm{M}} \sum_{\mathrm{y}=1}^{\mathrm{N}} \mathrm{I}(\mathrm{x}, \mathrm{y})(-\operatorname{InI}(\mathrm{x}, \mathrm{y}))
$$

\section{Abnormality Detection and Classification Via SVM}

SVM algorithm was introduced by Vapnik and Lerner (Vapnik, 1963) in 1963 and later on extended as Generalized Portrait algorithm by Vapnik and Chervonenkis (Vapnik \& Chervonenkis, 1964). This algorithm is based on the framework of statistical learning theory and termed as VC theory. It increases the generalization capability of learning machines to unseen data (Smola \& Schölkopf, 2004; Vapnik, 2000). In the past, SVM have provided excellent performance in different real-world applications including hand written digit recognition (Sch et al., 1997), object recognition (Pontil \& Verri, 1998), speaker identification (Wan et al., 2000), face detection in images (Osuna et al., 1997) and text categorization (Thorsten, 1999), to cite a few. This classification scheme is based on kernel methods (Bernhard \& Smola 2002; Hastie et al., 2009; Cristianini \& Shawe-Taylor 2000; Zhang et al., 2009).

In contrast to linear classification techniques, the kernel methods draw the original parameter vectors to a higher (possibly infinite) dimensional feature space via nonlinear kernel function. Here, the dot-products can efficiently be determined in higher dimensional space without computing the nonlinear mapping explicitly. The striking features that make SVM very attractive are the nonlinearly separable classes in which the original space can be linearly separated in the high dimensional feature space. Thus, SVM is able to solve complex nonlinear classification problems. Important characteristics of SVM lie in its ability to resolve classification problems using convex Quadratic Programming (QP) and the sparseness results from them. The learning is based on the principle of structural risk minimization. SVM tends to reduce the bound on the generalization error (error caused by learning machine on the test data which is not used for training) rather than minimizing the objective function which is based on the training samples (such as mean square error). Consequently, SVM performs well for the data outside the training set and enables the advantage of focusing on the training examples that is most difficult to categorize. These "borderline" training examples are called Support Vectors (SV).

Suykens has introduced Least Squares SVM (LS-SVM) (Suykens \& Vandewalle, 1999; Van Gestel et al., 2002) using the notion of Vapnik's SVM algorithm where the least squares term is inserted in the cost function. The variant circumvents is required to solve the complex QP problems consisting of a set of linear equations. This significantly reduces the problem complexity and makes the computation process efficient.

The hyper-planes in SVM technique define the decision boundaries by separating the data points of different types of pixels. SVM not only performs simple and linear classification, but also complex problems including non-linear one. Separable and non-separable problems are tackled according to their linear and nonlinear nature. The key concept of SVM is to plot the original data points from the input space to a higher or even infinite dimensional feature space to simplify the classification problem.

| A training dataset $\left(x_{i}, y_{i}\right)^{N}$ with $x_{i} \in R^{4}$, where $x_{i}$ Is the four input feature vector and $y_{i} \in\{-1,1\}$ represents the class labels of normal space and tumor brain space is considered. SVM's mapping from the four-dimensional input vector ( $\mathrm{x}$ ) space to the four-dimensional feature space is performed. The non-linear function is defined as $\varphi: \mathrm{R}^{4} \rightarrow \mathrm{R}^{4}$. Thus, the separated hyper-plane in the feature space is expressed as $\mathrm{w}^{\mathrm{T}} \varphi(\mathrm{x})+\mathrm{b}=0$, where $\mathrm{b} \in \mathrm{R}$ and $\mathrm{w}$ is an unknown vector having the same dimension as $\varphi(\mathrm{x})$. The data point $\mathrm{x}$ is allocated to the first class if $\mathrm{f}(\mathrm{x})=\operatorname{sign}\left(\mathrm{w}^{\mathrm{T}} \varphi(\mathrm{x})+\mathrm{b}=0\right)$ equals to +1 or to the second class if $\mathrm{f}(\mathrm{x})$ equals -1 .

The hyper-plane can be defined in different ways for linearly separable data. However, SVM depends on the maximum margin principle where the hyper-plane can be constructed with utmost distance between the two different types of pixels. Classification in SVM is initiated following the relations,

$$
\mid
$$

$$
\begin{array}{lll}
\mathrm{w}^{\mathrm{T}} \varphi\left(\mathrm{x}_{1}\right)+\mathrm{b} \geq+1 & \text { for } & \mathrm{y}_{\mathrm{i}}=+1 \\
\mathrm{w}^{\mathrm{T}} \varphi\left(\mathrm{x}_{1}\right)+\mathrm{b} \leq-1 & \text { for } & \mathrm{y}_{\mathrm{i}}=-1
\end{array}
$$

This is equivalent to,

1

Therefore, the classifier can be written as,

$$
\mathrm{y}_{\mathrm{i}}\left(\mathrm{w}^{\mathrm{t}} \varphi\left(\mathrm{x}_{\mathrm{i}}\right)+\mathrm{b}\right) \geq+1
$$




$$
\mathrm{f}(\mathrm{X})=\operatorname{sign}\left(\mathrm{w}^{\mathrm{T}} \varphi(\mathrm{x})+\mathrm{b}\right)
$$

Figure 9 illustrates the SVM classification using a hyper-plane, where the separating margin between the two types of pixels is minimized using data points denoted by $\mathrm{X}$ and $\mathrm{O}$. Here, the support vectors belong to the training set and reside on the hyper-planes boundary separating two classes.

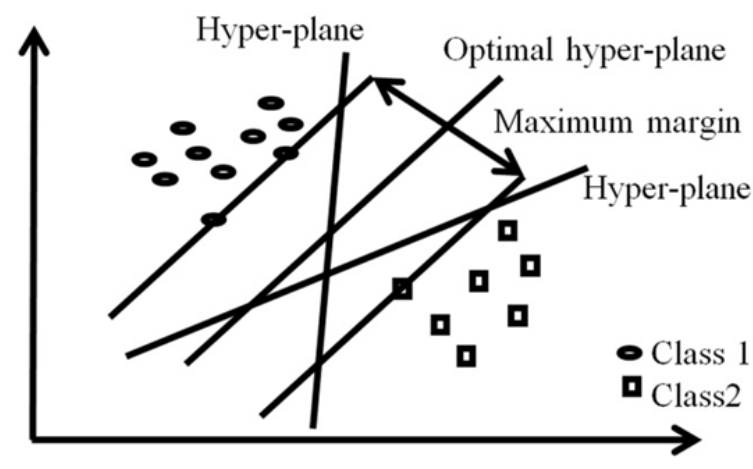

Figure 9. A separating hyper-plane (solid line) with maximum margin determined by linear SVM (Selvaraj et al., 2007)

\section{Results and Discussion}

\subsection{Dataset}

The proposed method is tested for the standard dataset called Internet Brain Segmentation Repository (IBSR) which is accessible at the Center for Morphometric Analysis, Massachusetts General Hospital (USA) as shown in (Figure 10) (NITRC, 2011). This being the most popular MRI segmentation database is extensively used in several studies (Gang et al., 2013; Ji et al., 2012; Balafar 2012; Eggert et al., 2012; Yousefi et al., 2012; Zhang et al., 2012; Iglesias et al., 2011; Hwang et al., 2011; Balafar et al., 2011; Rousseau et al., 2011; Somasundaram \& Kalaiselvi 2011; Alia et al., 2011; Ortiz et al., 2011; Tian et al., 2011; Bourouis \& Hamrouni, 2010; Sikka et al., 2009). The dataset (created in 1999) consists of 300 sliced MR images with 8 -bit gray scale images of $(256 \times 256)$ pixels. It contains multiple scans of a patient's tumor images and outlines which is acquired roughly in every six month intervals over a period of two and half years. Each series is stored in 60 slice image after conducting manual diagnosis and execution by well-trained experts (NITRC, 2011).

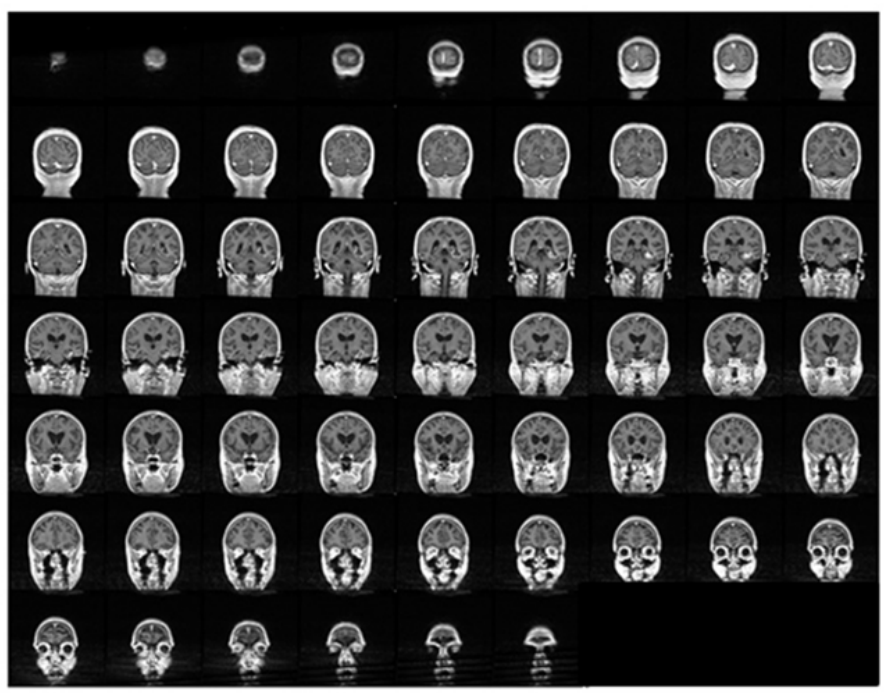

Figure 10. Three dimensional view of sixty slice images of the patient's living brain obtained from IBSR dataset.

\subsection{Feature Extraction}

SVM technique determines the abnormality in the training step, where the selection of feature is regarded as an important step (Abe, 2010). There exist 364 (91 portion $\times 4$ features) values of extracted features for each slice 
image. Therefore, for every 3D MR image a total of 21840 (364 features $\times 60$ slice) features value are classified using SVM method. The classification stage composed of training and testing phase. The feature vectors are comprised of approximate coefficients which are extracted from MR brain images and used as inputs for SVM. Besides, the distribution of these features is analyzed using three dimensional plots in which z-axis represents the normalized feature values while $\mathrm{x}$ and $\mathrm{y}$-axis characterizes the angular and distance span, respectively.

The distribution of the Mean of the divided slice images as displayed in Figure 11 exhibits the spreading of average values over the whole image with comparatively higher Mean in the tumor region than others. The results provide a clear indication concerning the Mean value of the non-tumor region. The value of Mean for tumor is observed to be about 160 .



Figure 11. Distribution of Mean in MR slice image.

The Standard Deviation as shown in Figure 12 reveal comparatively higher values in the tumor region than the non-tumor zone. Clearly, the values of Standard Deviation are not distributed over the entire image as in the case of Mean. The approximate value of the Standard Deviation in the tumor space is found to be greater than 70 .

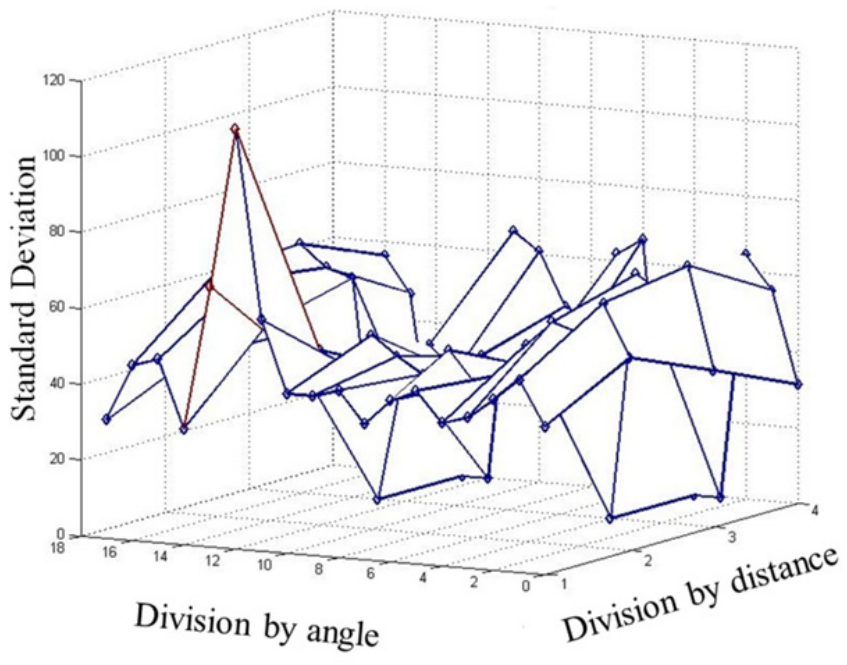

Figure 12. Distribution of Standard Deviation in MR slice image.

Figure 13 depicts the brain slice image Entropy distribution. They are distributed over the entire image with relatively higher occurrence in the tumor space than the non-tumor one. The Entropy value of the tumour space is discerned to be more than 500 . 


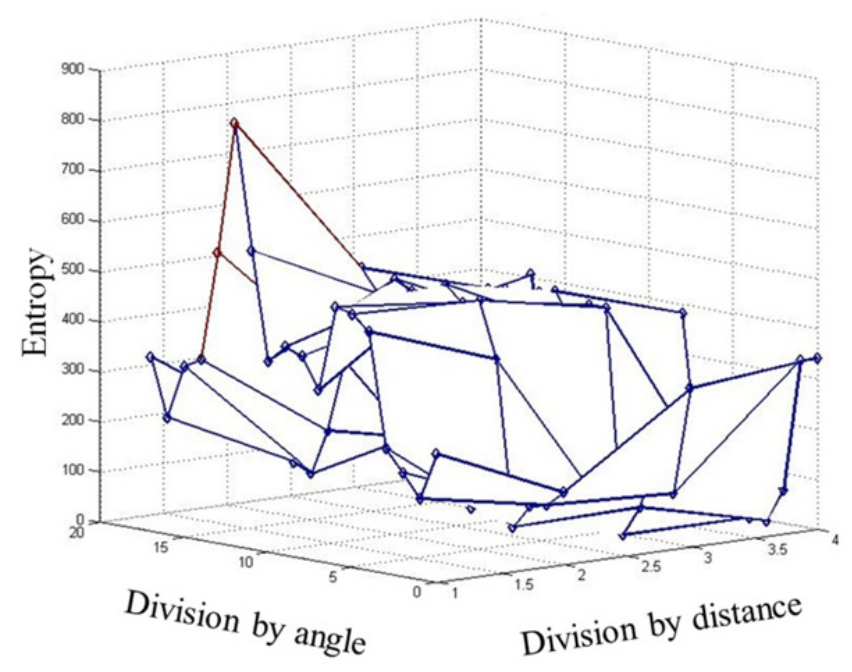

Figure 13. Distribution of Entropy in MR slice image.

Figure 14 showing the Energy distribution in the tumor space is found to be comparatively higher than the non-tumor one. Energy values are determined to be lower than 10.000 for the non-tumor space.



Figure 14. Distribution of Energy in MR slice image.

The generalized representations of the results are shown in Figure 15. The analysis is performed from the manual detection of tumor in MR slice image based on the relation between the Mean, Energy and Entropy. The brown color signifies the tumor space and blue one the non-tumor zone. 


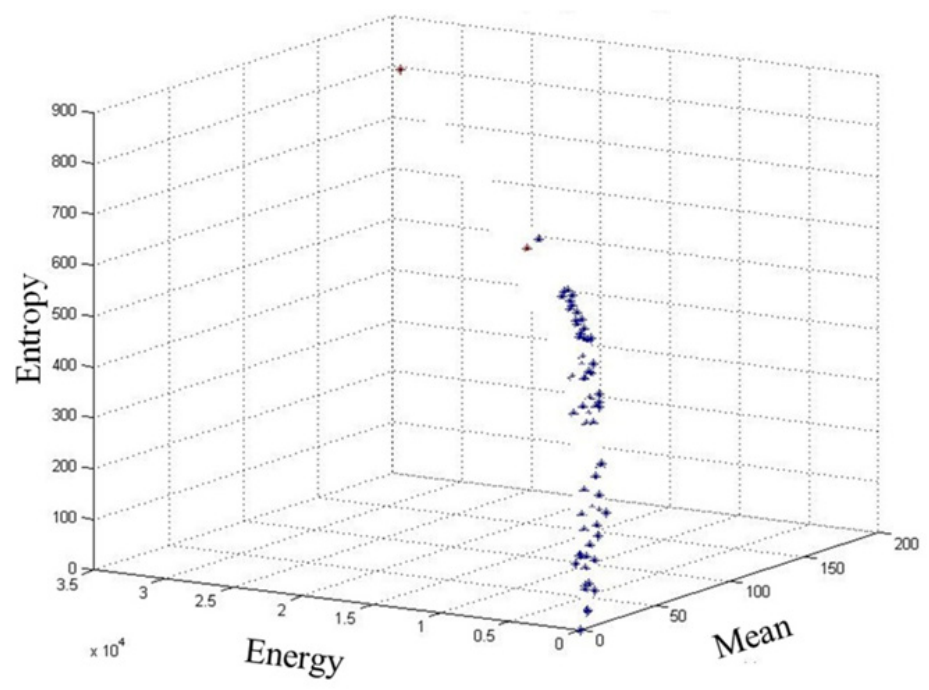

Figure 15. Relation among Mean, Energy and Entropy in MR slice image.

Figure 16 displays the overall comparison of Mean, Energy and Standard Deviation in the slice MR image. The values of Standard Deviation are observed to be distributed over the whole image. Furthermore, the Energy and the Mean values are found to reduce with the decrease of Standard Deviation for both tumor and non-tumor regions. The brown color symbolizes the tumor space and blue color for the non-tumor one.



Figure 16. Relation between Mean, Energy and Standard Deviation in MR slice image.

Practically, 180 slice images are used for training, and rest 120 for testing phase, from all the 300 slice images, 70 are abnormal and 230 is the normal one.

\subsection{Classification Results}

IBM SPSS Modeler Clementine 12.0 is used for classification (Khabaza \& Shearer, 1995; Ansheng, 2011). This being one of the influential, adaptable data and text analytical work-bench renders accurate predictive models quickly and intuitively, without programming. The data mining process can easily be visualized using this modeler's intuitive graphical interface. Firstly, the program is designed to model the classifier problem. Figure 17 displays the creation of model stream for SVM classification. 




Figure 17. Model stream for SVM classification.

Experiments are executed on MR images to verify the efficiency and robustness of the present classifier with the utilization of training and test data sets. The simulations are performed on a DELL PC Pentium with $3.4 \mathrm{GHz}$ processor and 16 GB RAM. The acquired features from MR images formed the input of SVM classifier. The formulation is carried out to ensure the adaptability and generalization of the classifiers. The first training set is found to be more biased towards the abnormal class. However, the second set having equal numbers of samples from both classes is used to achieve unbiased classifier training. Classification results obtained from with three MR images for training and two MR images for testing phases are listed in Table 1. We achieved an excellent average classification accuracy of $98.02 \%$ for training and $98.19 \%$ for testing.

Table 1. SVM classifier performance.

\begin{tabular}{ccc}
\hline IBSR Image Number & Training Accuracy & Testing Accuracy \\
\hline $536 \_32$ & $98.9 \%$ & $99.05 \%$ \\
$536 \_45$ & $98.2 \%$ & $98.49 \%$ \\
$536 \_47$ & $97 \%$ & $97.67 \%$ \\
$536 \_68$ & $98.45 \%$ & $98.26 \%$ \\
$536 \_88$ & $97.57 \%$ & $97.48 \%$ \\
\hline
\end{tabular}

\section{Conclusion}

A computer based technique using SVM classifiers is developed for automatic sorting of MR image slices corresponding to normal or abnormal cerebral tissues. The performances of the classifiers are analyzed in terms of statistical measures such as sensitivity, specificity and classification accuracy. The method consists of four steps such as cerebral tissue extraction, division, features categorization and classification. Threshold value and region of interest are determined using operator input and Otsu's algorithm. New brain slices image division through angular and distance span vectors are introduced. Following a histogram approach, the values of Standard Deviation, Mean, average Energy and Entropy are calculated for each merger space and compared with existing methods. Sixty slice images of the brain are from IBSR dataset are used. The proposed method achieves a value of average classification accuracy as much as $98.02 \%$ for training and $98.19 \%$ for testing. It is demonstrated that the new approach provides relatively higher performance than existing methods. Excellent features of the results suggest that the proposed technique can potentially be applied for medical image classification. Furthermore, it may contribute towards the development of computer aided intelligent health care systems. This automated analyses system can further be extended for the image classification with different pathological condition, types and disease status.

\section{Acknowledgements}

The author(s) would like to thank the Ministry of Higher Education \& Scientific Research-Iraq and Universiti 
Teknologi Malaysia for providing the financial support and facilities for this research.

\section{References}

Abe, S. (2010). Support Vector Machines for Pattern Classification. Springer.

Al-Badarneh, A., Najadat, H., \& Alraziqi, A. M. (2012). A Classifier to Detect Tumor Disease in MRI Brain Images. In Advances in Social Networks Analysis and Mining (ASONAM), 2012 IEEE/ACM International Conference. IEEE. IEEE, pp. 784-787. http://dx.doi.org/10.1109/ASONAM.2012.142

Alia, O. M., Mandava, R., \& Aziz, M. E. (2011). A Hybrid Harmony Search Algorithm for MRI Brain Segmentation. Evolutionary Intelligence, 4(1), 31-49. http://dx.doi.org/10.1007/s12065-011-0048-1

Al-Tamimi, M. S. H., \& Sulong, G. (2014a). A Review of Snake Models in Medical MR Image Segmentation. Jurnal Teknologi, 2(1), 101-106.

Al-Tamimi, M. S. H., \& Sulong, G. (2014b). Tumor Brain Detection Through MR Images: A Review of Literature. Journal of Theoretical and Applied Information Technology (JATIT), 62(2), 387-403.

Ansheng, L. (2011). Application Analysis of Clementine-Based Data Mining Algorithm. In 2011 International Conference on Intelligence Science and Information Engineering. IEEE., pp. 479-482. http://dx.doi.org/10.1109/ISIE.2011.76.

Antonie, L. (2008). Automated Segmentation and Classification of Brain Magnetic Resonance Imaging. C615 Project.

Ariffanan, M., \& Basri, M. (2008). Medical Image Classification and Symptoms Detection Using Neuro Fuzzy. $\mathrm{PhD}$ diss., Universiti Teknologi Malaysia, Faculty of Electrical Engineering.

Badran, E. F., Mahmoud, E. G., \& Hamdy, N. (2010). An Algorithm for Detecting Brain Tumors in MRI Images. In The 2010 International Conference on Computer Engineering \& Systems. IEEE, pp. 368-373. http://dx.doi.org/10.1109/ICCES.2010.5674887.

Balafar, M. A. (2012). Gaussian Mixture Model Based Segmentation Methods for Brain MRI Images. Artificial Intelligence Review, 429-439. http://dx.doi.org/10.1007/s10462-012-9317-3

Balafar, M. A., Ramli, A., \& Mashohor, S. (2011). Brain Magnetic Resonance Image Segmentation Using Novel Improvement for Expectation Maximizing. Neurosciences, 16(3), pp.242-247.

Bernhard, S., \& Smola, A. J. (2002). Learning With Kernels. MIT Pres, Cambridge, pp.73-76.

Bourouis, S., \& Hamrouni, K. (2010). 3D Ssegmentation of MRI Brain Using Level Set and Unsupervised Classification. International Journal of Image and Graphics, 10(1), 135-154.

Cawley, G. C., \& Talbot, N. L. C. (2004). Fast Exact Leave-One-Out Cross-Validation of Sparse Least-Squares Support Vector Machines. Neural Networks: The official journal of the International Neural Network Society, 17(10), 1467-1475. http://dx.doi.org/10.1016/j.neunet.2004.07.002

Chaplot, S., Patnaik, L.M., \& Jagannathan, N. R. (2006). Classification of Magnetic Resonance Brain Images Using Wavelets as Input to Support Vector Machine and Neural Network. Biomedical Signal Processing and Control, 1(1), 86-92.

Chen, S. (2004). Chaotic Spread Spectrum Watermarking for Remote Sensing Images. Journal of Electronic Imaging, 13(1), 146-165. http://dx.doi.org/10.1117/1.1631316

Chuang, C. C. et al. (2012). Patient-Oriented Simulation Based on Monte Carlo Algorithm by Using MRI Data. Biomedical engineering online, 11(1), p.21. http://dx.doi.org/10.1186/1475-925X-11-21

Clark, M.C. et al. (1994). MRI Seamentation Usina Fuzzy Clustering TechnGues. Engineering in Medicine and Biology Magazine, IEEE, 13(5), pp.730-742.

Cristianini, N., \& Shawe-Taylor, J. (2000). An Introduction to Support Vector Machines and Other Kernel-based Learning Methods First., United Kingdom, Cambridge: Cambridge University Press. http://dx.doi.org/10.1017/CBO9780511801389

Deepak, K. S. et al. (2013). An Efficient Approach To Predict Tumor In 2D Brain Image Using Classification Techniques. In Information Communication and Embedded Systems (ICICES), 2013 International Conference . IEEE. pp. 559-564.

Eggert, L. D. et al. (2012). Accuracy and Reliability of Automated Gray Matter Segmentation Pathways on Real and Simulated Structural Magnetic Resonance Images of the Human Brain Y. Fan, ed. Real and 
simulated structural magnetic resonance images of the human brain. PloS One, 7(9), 1-9. http://dx.doi.org/10.1371/journal.pone.0045081

El-Dahshan, E. A., Salem, A. B. M., \& Younis, T. H. (2009). A Hybrid Technique for Automatic MRI Brain Images Classification. Studia Univ. Babes-Bolyai, Informatica, 54(1), 55-67.

El-Dahshan, E. S. A., Hosny, T., \& Salem, A. B. M. (2010). Hybrid Intelligent Techniques for MRI Brain Images Classification. Digital Signal Processing, 20(2), 433-441. http://dx.doi.org/10.1016/j.dsp.2009.07.002

Ferlay, J. et al. (2010). GLOBOCAN 2008, Cancer Incidence and Mortality Worldwide: IARC CancerBase. Lyon, France: International Agency for Research on Cancer, 29(10).

Gang, Z. et al. (2013). An Unsupervised Method for Brain MRI Segmentation. International Journal of Emerging Technology and Advanced Engineering, 3(10), 8-13.

Goswami, S., \& Bhaiya, L. K. P. (2013). Brain Tumour Detection Using Unsupervised Learning Based Neural Network. In Communication Systems and Network Technologies (CSNT), 2013 International Conference. IEEE. IEEE., 573-577. http://dx.doi.org/10.1109/CSNT.2013.123

Hastie, T., Tibshirani, R., \& Friedman, J. (2009). The Elements of Statistical Learning Second Edi., New York USA: Springer.

Horská, A., \& Barker, P. B. (2010). Imaging of Brain Tumors: MR Spectroscopy and Metabolic Imaging. Neuroimaging clinics of North America, 20(3), 293-310. http://dx.doi.org/10.1016/j.nic.2010.04.003

Hwang, J., Han, Y. \& Park, H. (2011). Skull-Stripping Method for Brain MRI Using a 3D Level Set with a Speedup Operator. Journal of Magnetic Resonance Imaging, 34(2), 445-456. http://dx.doi.org/10.1002/jmri.22661

Ibrahim, W. H., Osman, A. A. A., \& Mohamed, Y. I. (2013). MRI Brain Image Classification Using Neural Networks. In Computing, Electrical and Electronics Engineering (ICCEEE), 2013 International Conference. IEEE. pp. 253-258. http://dx.doi.org/10.1109/ICCEEE.2013.6633943

Iglesias, J. E. et al. (2011). Robust Brain Extraction Across Datasets and Comparison With Publicly Available Methods. Medical Imaging, IEEE Transactions, 30(9), 1617-1634.

Improved, A., Gradient, C., \& Svm, L. S. (2005). An Improved Conjugate Gradient Scheme To The Slution of Least Squares SVM. Neural Networks, IEEE Transactions, 16(2), 498-501.

Jahne, B. (2005). Digital Image Processing 6th Editio., Springer-Verlag Berlin, germany.

Ji, Z. et al. (2012). Fuzzy Local Gaussian Mixture Model for Brain MR Image Segmentation. Information Technology in Biomedicine, IEEE Transactions, 16(3), 339-347.

Joshi, D. M., Rana, N. K., \& Misra, V. M. (2010). Classification of Brain Cancer Using Artificial Neural Network. In Electronic Computer Technology (ICECT), 2010 International Conference. IEEE. 112-116. http://dx.doi.org/10.1109/ICECTECH.2010.5479975

Khabaza, T., \& Shearer, C. (1995). Data Mining With Clementine. The digest of the IEEE Colloquium on Knowldge Discovery in Data-Base, Digest 1995 / 021 (B) - London, pp.1-5.

Klein, S. et al. (2010). Early Diagnosis of Dementia Based OnIntersubject Whole-Brain Dissimilarities. In Biomedical Imaging: From Nano to Macro, 2010 IEEE International Symposium. IEEE. $249-252$. http://dx.doi.org/10.1109/ISBI.2010.5490366

NITRC. (2011). NITRC: IBSR: Tool/Resource Info. Retrieved from http://www.nitrc.org/projects/ibsr

Noback, C. R. et al. (2005). The Human Nervous System Structure And Function Sixth Edit., New Jersey: Humana Press.

Nolte, J. (2013). The Human Brain in Photographs and Diagrams Fourth Edi., Elsevier Health Sciences.

Ortiz, A. et al. (2011). MR Brain Image Segmentation By Growing Hierarchical SOM and Probability Clustering. Electronics Letters, 47(10), 585-586. http://dx.doi.org/10.1049/el.2011.0322

Osuna, E., Freund, R., \& Girosit, F. (1997). Training Support Vector Machines: An Application to Face Detection. In Proceedings of IEEE Computer Society Conference on Computer Vision and Pattern Recognition. IEEE Comput. Soc., 130-136. http://dx.doi.org/10.1109/CVPR.1997.609310

Otsu, N. (1979). A Threshold Selection Method from Gray-Level Histograms. IEEE Transactions on Systems. Man and Cybernetics, 9(1), 62-66. 
Pontil, M., \& Verri, A. (1998). Support Vector Machines for 3D Object Recognition. IEEE Transactions on Pattern Analysis and Machine Intelligence, 20(6), 637-646. http://dx.doi.org/10.1109/34.683777

Rajini, N. H., \& Bhavani, R. (2011). Classification of MRI Brain Images Using k-Nearest Neighbor and Artificial Neural Network. In Recent Trends in Information Technology (ICRTIT), 2011 International Conference. IEEE. 563-568. http://dx.doi.org/10.1109/ICRTIT.2011.5972341

Rousseau, F., Habas, P. A., \& Studholme, C. (2011). Human Brain Labeling Using Image Similarities. In Computer Vision and Pattern Recognition (CVPR), 2011 IEEE Conference. 1081-1088.

Roy, S. et al. (2013). A Review on Automated Brain Tumor Detection and Segmentation from MRI of Brain. arXiv preprint arXiv, 1(1), 1-41.

Sadananthan et al. (2010). Skull Stripping Using Graph Cuts. NeuroImage, 49(1), 225-239.

Salankar, S. S., \& Bora, V. R. (2014). MRI Brain Cancer Classification Using Support Vector Machine. In Electrical, Electronics and Computer Science (SCEECS), 2014 IEEE Students' Conference. IEEE. 1-6. http://dx.doi.org/10.1109/SCEECS.2014.6804439

Sch, B. et al. (1997). Comparing Support Vector Machines with Gaussian Kernels to Radial Basis Function Classifiers. Signal Processing. IEEE Transactions, 45(11), 2758-2765.

Ségonne et al. (2004). A Hybrid Approach to the Skull Stripping Problem in MRI. NeuroImage, 22(3), 1060-1075.

Selvanayaki, K., \& Karnan, M. (2010). CAD System for Automatic Detection of Brain Tumor Through Magnetic Resonance Image-A Review. International Journal of Engineering Science and Technology, 2(10), $5890-5901$.

Selvaraj, H. et al. (2007). Brain MRI Slices Classification Using Least Squares Support Vector Machine. International Journal of Intelligent Computing in Medical Sciences \& Image Processing, 1(1), 21-33.

Sikka, K. et al. (2009). A Fully Automated Algorithm Under Modified FCM Framework for Improved Brain MR Image Segmentation. Magnetic Resonance Imaging, 27(7), 994-1004. http://dx.doi.org/10.1016/j.mri.2009.01.024

Smola, A. J., \& Schölkopf, B. (2004). A Tutorial On Support Vector Regression. Statistics and computing, 14(3), 199-222.

Somasundaram, K., \& Kalaiselvi, T. (2011). Automatic Brain Extraction Methods for T1 Magnetic Resonance Images Using Region Labeling and Morphological Operations. Computers in biology and medicine, 41(8), 716-725. http://dx.doi.org/10.1016/j.compbiomed.2011.06.008

Spisz, T. S., \& Bankman, I. N. (2000). Medical Image Processing and Analysis Software, Academic Press, Inc.

Suykens, J. A., \& Vandewalle, J. (1999). Least Squares Support Vector Machine Classifiers. Neural Processing Letters, 9(3), 293-300.

Technologies, C. (2014). Histogram Equalization for Image Enhancement Using MRI Brain Images. Computing and Communication Technologies (WCCCT), 2014 World Congress, IEEE, 80-83. http://dx.doi.org/10.1109/WCCCT.2014.45

Thorsten, J. (1999). Transductive Inference for Text Classification Using Support Vector Machines. ICML, 99, 200-209.

Tian, G. et al. (2011). Hybrid Genetic and Variational Expectation-Maximization Algorithm for Gaussian-Mixture-Model-Based Brain MR Image Segmentation. Information Technology in Biomedicine. IEEE Transactions, 15(3), 373-380.

Van Gestel, T. et al. (2002). Least Squares Support Vector Machines, Singapore: World Scientific.

Vapnik, V. (1963). Pattern Recognition Using Generalized Portrait Method. Automation and Remote Control, 24, 774-780.

Vapnik, V. (2000). The Nature of Statistical Learning Theory Second Edi. M. Jordan, ed., Springer-Verlag New York - USA.

Vapnik, V., \& Chervonenkis, A. (1964). A Note On Class of Perceptron. Automation and Remote Control.

Veloz, A., Orellana, A., \& Vielma, J. (2011). Brain Tumors: How Can Images and Segmentation Techniques Help? Diagnostic Techniques and Surgical Management of Brain Tumors, 67-92. 
Wan, V. et al. (2000). Support Vector Machines for Speaker Verification and Identification. Neural Networks Signal Process PROC IEEE, 2, 775-784.

Yousefi, S., Kehtarnavaz, N., \& Gholipour, A. (2012). Improved Labeling of Subcortical Brain Structures in Atlas-Based Segmentation of Magnetic. Biomedical Engineering. IEEE Transactions, 59(7), 1808-1817.

Zhang, H. et al. (2011). An Automated and Simple Method for Brain MR Image Extraction. Biomedical engineering online, 10(1), 81. http://dx.doi.org/10.1186/1475-925X-10-81

Zhang, N. et al. (2009). Multi-Kernel SVM Based Clasification For Brain Tumor Segmentation Of MRI Multi-Sequence. In Image Processing (ICIP), 2009 16th IEEE International Conference. IEEE. 3373-3376.

Zhang, T., Xia, Y., \& Feng, D. D. (2012). Clonal Selection Algorithm for Gaussian Mixture Model Based Segmentation of 3D Brain MR Images. Intelligent Science and Intelligent Data Engineering. pringer Berlin Heidelberg., 295-302.

\section{Copyrights}

Copyright for this article is retained by the author(s), with first publication rights granted to the journal.

This is an open-access article distributed under the terms and conditions of the Creative Commons Attribution license (http://creativecommons.org/licenses/by/3.0/). 